\title{
Preparation and Electrochemical Testing of Polyaniline (PANI) Nanoparticles with Uniform Morphology and Good Dispersion in a Water-task-specific Ionic Liquid Medium
}

\author{
Dingjun ZHANG ${ }^{1}{ }^{*}$, Yuxian CHEN ${ }^{1}$, Xue BAI ${ }^{1}$, Xianwen LI $^{2}$, Yingxia MA ${ }^{1}$, Zhenbin CHEN ${ }^{1}$ \\ ${ }^{1}$ State Key Laboratory of Advanced Processing and Recycling of Non-ferrous Metals, Lanzhou University of Technology, \\ Lanzhou 730050, China \\ ${ }^{2}$ Petro China Chang-qing Oilfield Company Xi'an, 710021 China
}

cross $^{\text {ref }}$ http://dx.doi.org/10.5755/j01.ms.25.3.19145

Received 30 September 2017; accepted 30 March 2018

\begin{abstract}
Polyaniline (PANI) nanoparticles with uniform morphology and good dispersion were prepared by using the task-specific Ionic Liquid (IL) 1-ester-3-methyl-imidazolium tetrafluoroborate ([EAMIM]BF4) as a soft template by chemical oxidation in a water-IL medium. The morphological, structural and electronic properties of polyaniline (PANI) products, controlled by the IL $[\mathrm{EAMIM}] \mathrm{BF}_{4}$, were synthesized and investigated. Products were characterized by SEM, FT-IR and the measurements of conductivity. The addition of [EAMIM]BF4 strongly influenced the morphology, uniformity, and conductivity of the PANI. Depending on the [EAMIM]BF 4 :ANI ratio, various nanostructures of PANI could be prepared and the dominant morphologies are the rod-like and fiber-like elongated structures under most experimental conditions. Samples prepared in the presence of IL [EAMIM]BF4 show uniform morphology, good dispersion and good electrochemical performance. At the current density of $0.5 \mathrm{~A} / \mathrm{g}$, the specific capacitance of PANI was $396 \mathrm{~F} \cdot \mathrm{g}^{-1}$. Compared with the PANI without ionic liquids, the specific capacitance was increased by $165 \mathrm{~F}_{\mathrm{g}} \mathrm{g}^{-1}$.

Keywords: PANI, ionic liquid, morphology, electrochemical performance.
\end{abstract}

\section{INTRODUCTION}

Polyaniline (PANI) nanoparticles have received a great amount of attention, because of its straight-forward and cost-effective synthesis process, excellent mechanical properties, thermal stability and helpful electrical properties [1-3], which can be controlled easily by doping proton or changing the state of its oxidation. The size and shape of PANI nanoparticles [4] are crucial in various applications, including conductive blends and nanocomposites which are used to reaction catalysts. One-dimensional PANI nanostructures [5], like nanofibers, have particular potential to be used in many domains, like nanocomposites, coatings, catalytic supports, actuators, etc.

More recently, research has focused on enhancing the properties of PANI by developing more various nanostructures. In order to acquire different morphologies of PANIs, many different synthetic routes have been developed. The most similar are produced from routes, which contain hard templates, soft templates $[6,7]$ and polymer additives acting as directing agents of the structure. The method of the soft template is based upon the selfassembly of a monomer that is driven by the molecules of the soft template and is controlled by the chemical bonding except the covalent bonding. The most frequently used soft templates are surfactants, which form with aniline salts that self-assemble into micelles. By changing the concentration of the surfactant [8-13], the micelles are endowed more morphologies, such as spherical, cylindrical and lamellar.
Ionic liquids (ILs) is a type of organic salt and it is liquid when the temperature is at or near room temperature. These compounds have got more attention because of their remarkable properties [14-16]. In water, the behave of ILs like typical surfactants, because they form micelles under the concentration above the critical micellar concentration [17]. Howbeit, compared with typical conventional surfactants, there are some differences amongst them because they have only a short hydrophobic chain [18-20]. Additionally, conductivity measurements of ILs, under aqueous solutions, indicate that IL molecules aggregate together when the concentrations below the critical micellar concentration. At higher concentrations, it is possible to form disk-like structures, which have a distinct long-range order [21]. ILs show intensive trend towards selforganization and can form congeries, which can selfassemble into three-dimens-ional networks [22], which can act as the soft templates to form polymer nanoparticles.

Due to these unique properties, ILs have attracted increasing attention in many areas, like chemical synthesis, polymerization, and catalysis. Recently, ILs was applied as an additive during the chemical oxidative polymerization of aniline (ANI). They form larger nanostructures through hydrogen bonding in the liquid state, which is of high interest in the preparation of various nanoparticles [23].

A striking feature lies in that the type and nature of IL used for PANI synthesis that profoundly affect the morphology of PANI and their physical properties can be fine-tuned significantly by manipulation of their morphology in water-IL media.

\footnotetext{
${ }^{*}$ Corresponding author. Tel.: +86 13609321215.
}

E-mail address: zhangdingjun@lut.cn (D.J. Zhang) 
A primary investigation undertaken in the authors' laboratory has revealed that PANI nanoparticles with various morphologies can be synthesized successfully by using the ILs with different molecular structures as soft templates by chemical oxidation in a water-IL medium. It can be feasible to realize such morphology manipulation of the PANI by modifying the choice of ILs. This would, in turn, make it possible to tune and further improve the physical properties of the PANI nanoparticles. Therefore, chemical oxidative polymerization of ANI in water-IL media has been attracting increased attention as a promising route to synthesize the PANI nanoparticles with enhanced properties [24].

In the present study, the preparation of PANI nanoparticles with uniform morphology and good dispersion was investigated using the task-specific IL [EAMIM]BF4 as soft template by the chemical oxidation in a water-IL medium. The morphology of the PANI nanoparticles was monitored using SEM, the chemical structure was studied by the FT-IR, their constant current charge-discharge performance and the cyclic voltammetry (CV) curves of the electrode materials were tested using a CHI660C.

\section{EXPERIMENTAL}

\subsection{Chemicals}

The chemicals used included: aniline (ANI; Connell Co. Ltd; AR), ammonium persulfate (APS, Yantai SS Chemical Co. Ltd; AR), 1-Methylimidazole $\left(\mathrm{C}_{4} \mathrm{H}_{6} \mathrm{~N}_{2}\right.$; Shenzhen jin feng industrial Co. LTD; AR), acetone $\left(\mathrm{CH}_{3} \mathrm{COCH}_{3}\right.$; Beijing Chemical Works; AR), absolute ethyl alcohol $\left(\mathrm{C}_{2} \mathrm{H}_{6} \mathrm{O}\right.$; Tianjin Fuyu Chemical Co. Ltd; AR), acetonitrile $\left(\mathrm{C}_{2} \mathrm{H}_{3} \mathrm{~N}\right.$; Beijing chemical Works; AR), xylol $\left(\mathrm{C}_{8} \mathrm{H}_{10}\right.$; Yantai SS Chemical Co. Ltd; AR), concentrated sulfuric acid $\left(\mathrm{H}_{2} \mathrm{SO}_{4}\right.$; Shenyang Minlian Chemical Co. Ltd; 98\%), n-butyl bromide $\left(\mathrm{C}_{4} \mathrm{H}_{9} \mathrm{Br}\right.$; Tianjin Yuanli Chemical Co. Ltd; AR), 1.3-propane sultone $\left(\mathrm{C}_{3} \mathrm{H}_{6} \mathrm{O}_{3} \mathrm{~S}\right.$; Shanghai Research and Development of Biology CO. Ltd; AR), 2chloroethanol $\left(\mathrm{C}_{2} \mathrm{H}_{5} \mathrm{ClO}\right.$; Shanghai Fuzhe Chemical Co. Ltd; AR), ethyl chloroacetate $\left(\mathrm{C}_{4} \mathrm{H}_{7} \mathrm{ClO}_{2}\right.$; Shanghai Zhongqin Chemical Reagent Co. Ltd; AR), sodium fluoride $\left(\mathrm{NaBF}_{4}\right.$; Shanghai Zhongqin Chemical Reagent Co. Ltd; AR), ethyl acetate $\left(\mathrm{CH}_{3} \mathrm{COOC}_{2} \mathrm{H}_{5}\right.$; Lee Long Bohua (Tianjin) chemical pharmaceutical company; AR) and distilled water $\left(\mathrm{H}_{2} \mathrm{O}\right.$; Self-control).

\subsection{Polymerization procedure}

\subsubsection{The synthesis of PANI nanoparticles}

Standard procedure of polymerization was as follows:

The new distilled aniline and the target IL [EAMIM]BF4 was dispersed successively in a certain amount of distilled water, and weighed amounts of APS were scattered uniformly in distilled water. After 15 min of ultrasonic dispersion, they were cooled in an ice bath with $0-5{ }^{\circ} \mathrm{C}$. After $30 \mathrm{~min}$, the aqueous solution of APS in ANI was added slowly to the mixed liquid of IL to aniline, and was reacted for $24 \mathrm{~h}$ under $0-5^{\circ} \mathrm{C}$. The greenish-black PANI nanoparticle product was collected after suction filtering, washing and drying about $48 \mathrm{~h}$ under $60{ }^{\circ} \mathrm{C}$, the mole ratios of ANI to ionic liquids respectively were $4: 1$, $2: 1,1: 1$, and $1: 2$.

\subsubsection{Synthesis of the ionic liquid}

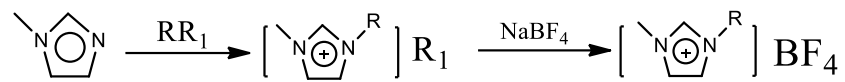

$$
\begin{aligned}
& \mathrm{R}=1 \text {-ethyl acetate; } \mathrm{R}_{1}=-\mathrm{Cl}
\end{aligned}
$$

Fig. 1. The synthetic routes of four kinds of ionic liquids

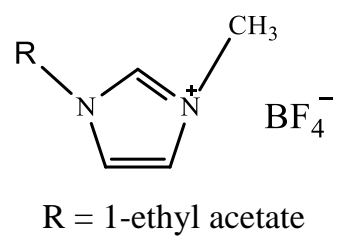

Fig. 2. The molecular structure of the target compounds

The ionic liquid with various groups was synthesized and their synthetic routes are presented in Fig. 1. Meanwhile, the molecular structure of the target compounds is shown in Fig. 2. The procedure of polymerization was as follows:

Weighed amounts of 1-methylimidazole were dissolved in a certain amounts of acetone in a nitrogen atmosphere at $55{ }^{\circ} \mathrm{C}$ with constant magnetic stirring. Ethyl acetate was slowly added to the above mixture and was stirred for approximately $24 \mathrm{~h}$ at $75^{\circ} \mathrm{C}$. The intermediate of the IL, was isolated by filtration, washed thoroughly by ethanol and dried at $75^{\circ} \mathrm{C}$ under vacuum.

Weighed amounts of the intermediate were dissolved in a certain amounts of acetonitrile with constant stirring. When dissolved completely, boron sodium fluoride was dropped to the above mixture. After stirring for approximately $24 \mathrm{~h}$ under a room temperature, a mixture of yellow liquid and white solid was obtained. The viscous yellow liquid product, the objective IL, was isolated by filtration, washed thoroughly and dried using rotary evaporation and vacuum at $75^{\circ} \mathrm{C}$ for approximately $24 \mathrm{~h}$.

\subsection{Characterization}

\subsubsection{Characterization of structure}

The morphology of PANI observed by SEM. After ultrasonic dispersion in the ethanol, the PANI powder was dropped onto a copper cylinder. The morphology of the sample was observed by a JEOL 6700F scanning electron microscope.

\subsubsection{Electrochemical characterization tests}

The constant current charge-discharge performance and cyclic voltammetry (CV) curves for the electrode materials were tested using a CHI660C manufactured by $\mathrm{CH}$ Instruments, China. In this test, the working electrode was PAn, a sheet of platinum $1 \mathrm{~cm} \times 1 \mathrm{~cm}$ was the auxiliary electrode, and the reference electrode was a saturated calomel electrode. The electrolyte was $1 \mathrm{~mol} / \mathrm{L} \mathrm{H} 2 \mathrm{SO} 4$.The specific capacitance of PANI could be calculated using the following formula :

$$
C=l \times \frac{\Delta t}{(\Delta V \times m)},
$$


where: $C$ is the symbol of mass ratio $\left(\mathrm{F}_{\mathrm{g}} \mathrm{g}^{-1}\right) ; I$ represent capacitance discharge current (A), $m$ is the quality of the active electrode $(\mathrm{g}), \Delta t$ is the discharge time and the discharge voltage drop is represented by $\Delta V$.

The total weight of the electrode material was $8 \mathrm{mg}$. The proportions of the mixture were $80 \mathrm{wt} . \%$ of PANI-C with 7.5 wt. $\%$ of graphite and 7.5 wt.\% of acetylene. After mixing evenly, the electrode mix was dropped into PTFE emulsion with a mass fraction of $5 \%$, and a few drops of ethanol. The electrode paste was smeared on the processed stainless steel wire mesh over an area of about $1 \mathrm{~cm}^{2}$. The stainless steel net then was subjected to a pressure of $10 \mathrm{MPa}$ and put into an oven to dry for $16 \mathrm{~h}$ at $60{ }^{\circ} \mathrm{C}$.

\section{RESULTS AND DISCUSSION}

The IL [EAMIM]BF4 was used to study the effects of IL on PANI properties and morphology. In order to discuss the effects of the ANI/ionic liquid (ANI/IL) molar ratio on the morphology of the PANI, four mole ratios: 4:1, 2:1, 1:1, and $1: 2$, were investigated. Because experimental

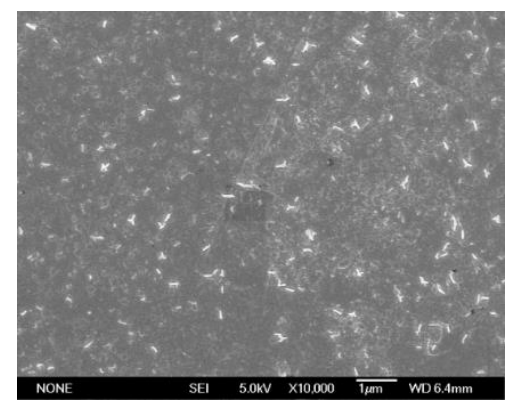

a

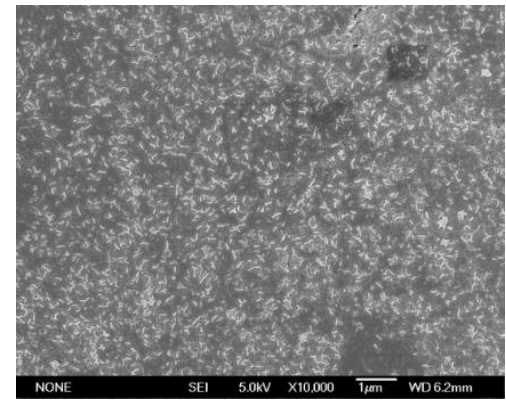

c conditions were same for all of the tests and only the mole ratio of ANI to IL was varied, it could be concluded that the IL was the only factor for the observed differences in morphology of the prepared PANIs.

\subsection{The effect of the dosage of [EAMIM]BF 4 on the morphology of PANI}

SEM micrographs of PANI prepared under different mole ratios of AN to [EAMIM]BF4 ionic liquid are shown in Fig. 3. When the molar ratio of AN to the [AMIM]BF4 ionic liquid was maximum (Fig. 3 a), the PANI exhibited a dispersed rod-like nanostructure with lower crystalline productivity and density. With increasing the mole fraction of ionic liquid (Fig. $3 \mathrm{~b}$ and c), the produced volume and density of the product were better. However, when the mole ratio of $\mathrm{AN}$ to the [AMIM]BF4 ionic liquid was minimum (Fig. $3 \mathrm{~d}$ ), the produced volume and product density became unsatisfactory, though the special morphology of the product did not disappear.

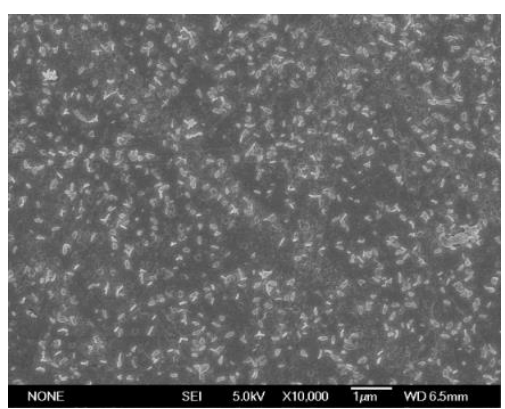

b

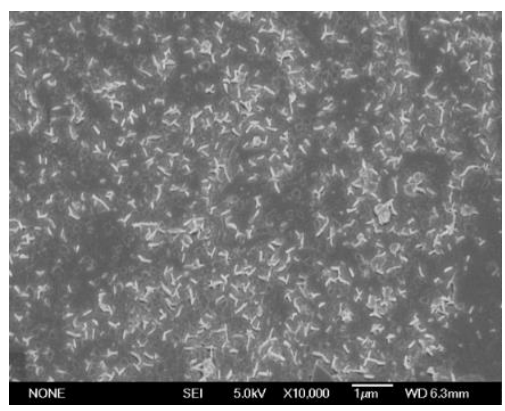

d

Fig. 3. SEM of PANI prepared under different ratios of $n_{\text {An: }} n_{I L}: a-4: 1 ; b-2: 1 ; c-1: 1 ; d-1: 2$

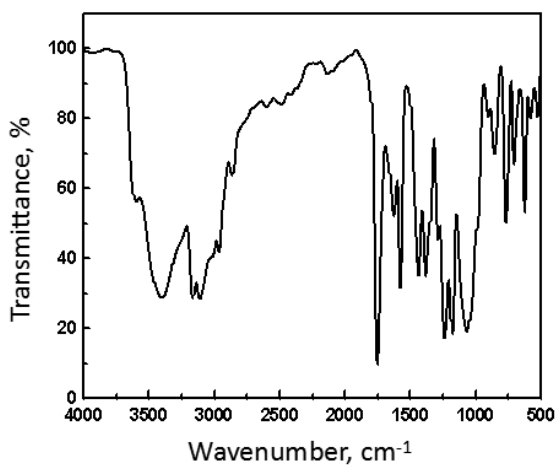

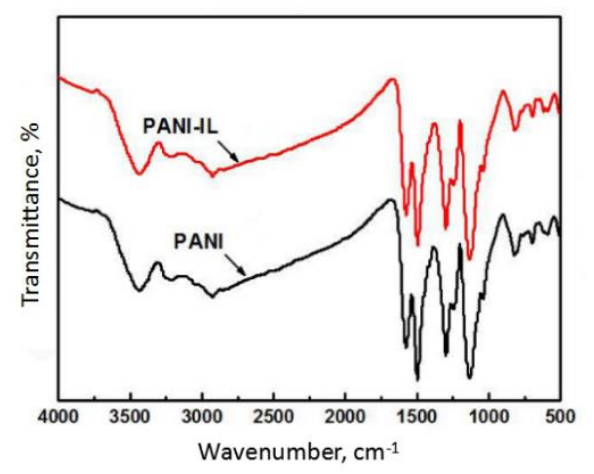

b

Fig. 4. The FT-IR spectra for the $\left[\mathrm{EAMIM}^{\mathrm{B}} \mathrm{BF}_{4}\right.$ ionic liquid and the PANI 
Through these SEM micrographs (Fig. 3), it is evident that under different molar ratios, although yield and density of the product differ, the PANI was still presented a dispersed rod-like nanostructure, i.e. the morphology of the PANI was strictly controlled by the [EAMIM]BF4 ionic liquid.

\subsection{The FT-IR Spectrum}

\subsubsection{FT-IR}

The IR spectra for [EAMIM]BF4 ionic liquid is presented in Fig. 4 a. The peaks at $3166 \mathrm{~cm}^{-1}$ and $1575 \mathrm{~cm}^{-1}$ respectively correspond to $\mathrm{C}-\mathrm{H}$ bond stretching vibration and the skeleton vibration of the imidazole ring, while the peaks at $850 \mathrm{~cm}^{-1}$ and $762 \mathrm{~cm}^{-1}$ have been assigned to outof-plane bending vibrations of the $\mathrm{C}-\mathrm{H}$ bonds in the imidazole ring. The bands at $2967 \mathrm{~cm}^{-1}$ and $1375 \mathrm{~cm}^{-1}$ respectively belong to saturated $\mathrm{C}-\mathrm{H}$ stretching deformation and bending vibrations, while the peaks at $1757 \mathrm{~cm}^{-1}$ and $1177 \mathrm{~cm}^{-1}$ respectively correspond to $\mathrm{C}=\mathrm{O}$ stretching vibration of the ester group and the $\mathrm{C}-\mathrm{O}$ stretching vibration of the ester group, attesting that there are ester groups in the ionic liquid. The peak at $1063 \mathrm{~cm}^{-1}$ is associated with the BF stretching vibration, and the band at $3405 \mathrm{~cm}^{-1}$ has been assigned to $\mathrm{O}-\mathrm{H}$ stretching vibration, which illustrates that the ionic liquid has some impurity water because of the hydroscopicity of the boric acid radical ion. From the above can be concluded that the ionic liquid is [EAMIM]BF4.

The FT-IR spectrum of PANI samples before and after adding the [EAMIM]BF4 ionic liquid is shown in Fig. 4 b. The band at $3442 \mathrm{~cm}^{-1}$ is attributable to $\mathrm{N}-\mathrm{H}$ stretching vibrations, while the peaks at $1580 \mathrm{~cm}^{-1}$ and $1502 \mathrm{~cm}^{-1}$ can be attributed to $\mathrm{C}=\mathrm{C}$ stretching deformations of the PANI quinonoid and benzenoid rings, respectively. The band at $1295 \mathrm{~cm}^{-1}$ is attributable to $\mathrm{C}-\mathrm{N}$ stretching vibrations of the PANI benzenoid rings, the band at $1137 \mathrm{~cm}^{-1}$ is characteristic of the doped state of PANI [25] and the peak at $813 \mathrm{~cm}^{-1}$ can is attributable to $\mathrm{C}-\mathrm{H}$ out-of-plane bending in the 1,2-disubstituted aromatic rings.

Very similar features have been shown by the FT-IR spectra, which indicate that the addition of [EAMIM]BF4 ionic liquid did not alter significantly the chemical composition or structure of the PANI.

\subsection{The electrochemical performance of PANI}

\subsubsection{Effect of PANI preparation conditions on the CV curves}

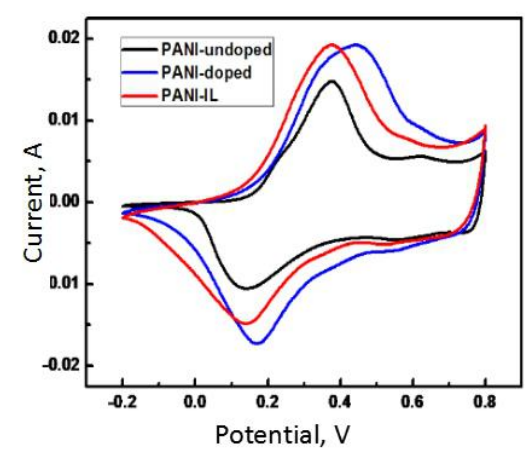

Fig. 5. Cyclic voltammetry curves for PANI as an electrode prepared under different conditions
The CV curves for PANI as the electrode prepared in $1 \mathrm{~mol} / \mathrm{L} \mathrm{H} 2 \mathrm{SO} 4$ under three different conditions are shown in Fig. 5. The PANI was doped using $1 \mathrm{~mol} / \mathrm{L} \mathrm{HCl}$ and the PANI-IL was controlled by [EAMIM]BF4 ionic liquid. It can be observed that every $\mathrm{CV}$ curve exhibited a significant redox peak, while the potential difference of the peak of non-doped PANI was $0.24 \mathrm{~V}$, for doped PANI was $0.28 \mathrm{~V}$ and for PANI controlled by ionic liquid was $0.22 \mathrm{~V}$.

It can be concluded that PANI controlled by the ionic liquid was better and the area of the CV curve of the PANI prepared using the ionic liquid as the structural control agent was largest, which means that the quality-specific capacitance of the PANI was optimum. Because the uniform morphology of the PANI was controlled by the ionic liquid, its electrochemical performance was improved.

\subsubsection{Effect of Scanning rate on CV curves of PANI}

The CV curves for PANI electrodes at different scanning rates are shown in Fig. 6. Both of the CV curves show a pair of significant characteristic redox peaks, which indicates that both of the PANI samples show reversibility of the redox reaction under two different scanning rates.

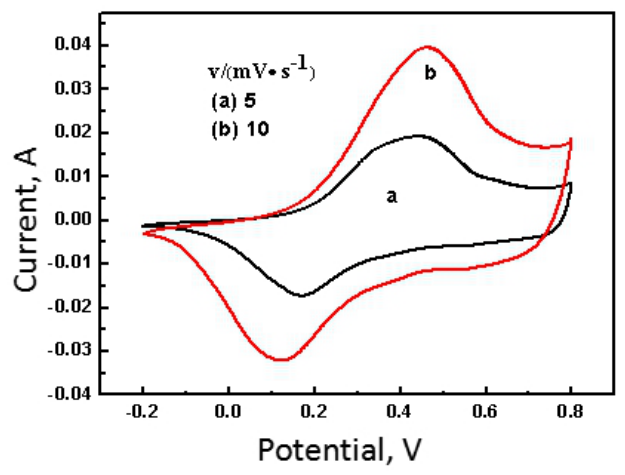

Fig. 6. Cyclic voltammetry curves for PANI electrodes at different scanning rates: $\mathrm{a}-5 \mathrm{mV} . \mathrm{s}^{-1} ; \mathrm{b}-10 \mathrm{mV} . \mathrm{s}^{-1}$

However, the potential difference of CV curve of the $b$ test condition was greater than that of the test, which indicates that the redox reversibility under the ' $a$ ' condition was better than for the ' $b$ ' condition. Furthermore, the area bounded by the $\mathrm{CV}$ curve under the ' $\mathrm{b}$ ' test condition was clearly larger than that pf the ' $a$ ' curve, which is indicative that the specific capacitance under the ' $b$ ' test condition was better than for the ' $a$ ' condition.

\subsubsection{PANI Charging and discharging test}

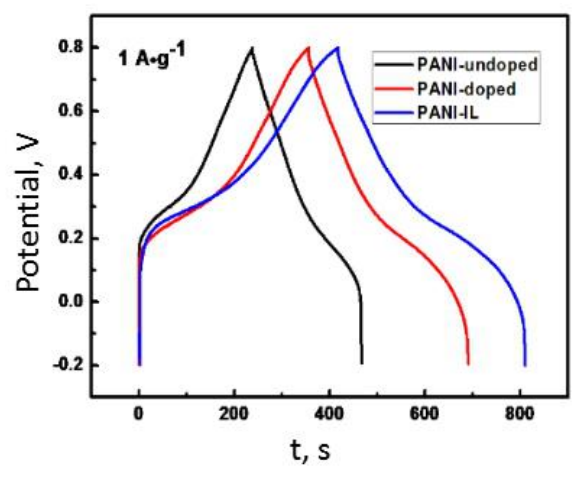

Fig. 7. Constant current charge/discharge curves for PANI as an electrode prepared under different conditions 
The charge/discharge figure for PANI, as a capacitor prepared under different coditions when the current density is $1 \mathrm{~A}^{-1} \mathrm{~g}^{-1}$, is supplied in Fig. 7. PANI doped by $1 \mathrm{~mol} / \mathrm{L} \mathrm{HCl}$ and the PANI-IL was controlled by the [EAMIM]BF4 ionic liquid. It can be observed that every curve exhibited a symmetrical triangle, which indicates the performance of electrochemical capacitance of PANI prepared under these three different conditions was better. The specific capacitance of the PANI can be calculated using Eq. 1 .

It was calculated that the capacitance of non-doped PANI was $231 \mathrm{~F}^{-1}$, that of the doped PANI was $338 \mathrm{~F}^{-g^{-1}}$, and that of the PANI controlled by the ionic liquid was $396 \mathrm{~F}^{-1}{ }^{-1}$, which verified the conclusion from the capacitance tests indicating that PANI is best-controlled by the ionic liquid.

It is reported that the feed ratio of aniline to different templates played a very important role in constructing the hierarchically nano-structure, which would, hence, determine the electrochemical erformance of the materials. Using the template-assisted strategy and controlling the feed ratio of aniline to templates, the nanometer structure of nano-PANI was obtained [26].

PANI samples synthesised in the presence of [EAMIM]BF4 had uniform structure than the corresponding PANI synthesised in Qingbo Yu et al [27]. (2015).

This is because PANI samples, when the mole ratio of [EAMIM]BF4 to ANI is 1, have uniform structure (Fig. $3 \mathrm{c}$ ) and, consequently, exhibit the electrochemical performance. Therefore, the presence of [EAMIM]BF4 can be used to optimise the reaction conditions for obtaining PANI products with uniform structure and excellent electrochemical performance.

\subsubsection{Effect of current density on the charging and discharging of PANI}

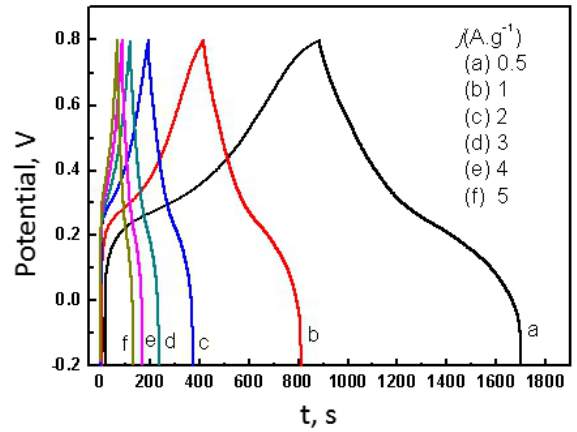

Fig. 8. Constant current charge/discharge curves for PANI at various current densities

The charge/discharge curves under constant current, for PANI at various current densities are presented in Fig. 8 from Eq. 1.

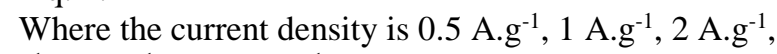
$3 \mathrm{~A} \cdot \mathrm{g}^{-1}, 4 \mathrm{~A} \cdot \mathrm{g}^{-1}$, and $5 \mathrm{~A} \cdot \mathrm{g}^{-1}$, and the specific capacitances of the PANI was $422 \mathrm{~F}_{\mathrm{g}} \mathrm{g}^{-1}, 396 \mathrm{~F} \cdot \mathrm{g}^{-1}, 384 \mathrm{~F} \cdot \mathrm{g}^{-1}, 372 \mathrm{~F} \cdot \mathrm{g}^{-1}, 340$ F.g ${ }^{-1}$, and 335 F.g ${ }^{-1}$, respectively.

It was thus confirmed that the specific capacitance value of PANI decreased with increasing the current density, though this could have been effect of polarization. Under a larger current-density conditions, when the speed of the cell response could not satisfy the demand of the electrochemical reaction, hydrogen diffuses to the electrode/electrolyte interface, the capacitance is reduced.

\section{CONCLUSIONS}

The IL [EAMIM]BF4 influences the morphology of the PANI. As overall test was maintained notionally constant throughout all of the polymerization of ANI and only the mole ratio of ANI to IL was changed. It could be determined that the addition of IL was the only factor for the different morphologies of the prepared PANI. This was confirmed by the SEM spectra which show significant differences in morphology of the PANIs. The best-defined nanoparticles were prepared with [EAMIM]BF4 additions to the ANI:IL mixture at a mole ratio of $1: 1$. Under these conditions the particles were dispersed evenly and had a special homogeneous rod-like nanostructure with a diameter of approximately $50 \mathrm{~nm}$ and length of approximately $100 \mathrm{~nm}$.

From the electrochemical performance of the PANI, it could be deduced that a uniform structure of the PANI was favorable to improve its electrochemical properties.

Therefore, the presence of [EAMIM]BF4 can be used to optimise the reaction conditions for obtaining PANI products with uniform structure and excellent electrochemical performance.

\section{Acknowledgments}

The authors are grateful to the National Natural Science Foundation of China (NSFC, 51663013).

\section{REFERENCES}

1. Sonker, R.K., Yadav, B.C., $\quad$ Dzhardimalieva, G.I. Preparation and Properties of Nanostructured PANI Thin Film and Its Application as Low Temperature $\mathrm{NO}_{2}$ Sensor Journal of Inorganic and Organometallic Polymers and Materials 26 (6) 2016: pp. $1428-1433$. https://doi.org/10.1007/s10904-016-0439-y

2. Somani, P.R. Synthesis and Characterization of Polyaniline Dispersions Materials Chemistry \& Physics 77 (1) 2002: pp. 81. https://doi.org/10.1016/s0254-0584(01)00579-x

3. Fu, Z.B. Preparation and Modification of Polyaniline and Its Interaction with Hydrogen Beijing: China Academy of Engineering Physics 2009: pp. 782-788.

4. Tan, Y.T., Ran, F., Wang, L.R. Synthesis and Electrochemical Properties of Hollow Polyaniline Microspheres by a Sulfonated Polystyrene Template Journal of Applied Polymer Science 127 2013: pp. 1544-1549. https://doi.org/10.1002/app.36781

5. Wang, H.Y., Li, L., Zhang, L., Ran, F. The Unique Morphology Role of Thorn Surface in Determining Electrochemical Performance of Polyaniline Nano-fibers via One-step Method Journal of Applied Polymer Science 132 (28) 2015: pp. 1-6. https://doi.org/10.1002/app.42266

6. Yu, X.F., Li, Y.X., Kalantar-zadeh, K. Synthesis and Electrochemical Properties of Template-based Polyaniline Nanowires and Template-free Nanofibril Arrays: Two Potential Nanostructures for Gas Sensors Sensors and Actuators B: Chemical 136 (1) 2009: pp. 1-7. https://doi.org/10.1016/j.snb.2008.10.068

7. Pahovnik, D., Zagar, E., Kogej, K. PANI Nanostructures 
Prepared in Acidic Aqueous Solutions of Ionic Liquids Acting as Soft Templates European Polymer Journal 49 2013: pp. $1381-1390$.

https://doi.org/10.1016/j.eurpolymj.2013.02.019

8. Li, R., Zhang, X.F. Preparation and Characterization of Polyaniline Micro/nanotubes by Soft Template Fine Chemicals 25 (4) 2008: pp. 321-328. https://doi.org/10.1016/j.synthmet.2010.01.022

9. Jung, W. H., Lee, Y. M., McCarthy, S. P. Synthesis of Needle-like Polyanilines Journal of Vinyl and Additive Technology 13 (2) 2007:pp. 76-86. https://doi.org/10.1002/vnl.20106

10. Kubisa, P. Application of Ionic Liquids as Solvents for Polymerization Processes Progress in Polymer Science 29 (1) 2004: pp. 3-12. https://doi.org/10.1016/j.progpolymsci.2003.10.002

11. Pahovnik, D., Zagar, E., Kogej, K. Polyaniline Nanostructures Prepared in Acidic Aqueous Solutions of Ionic Liquids Acting as Soft Templates European Polymer Journal 49 2013: pp. 1381-1390.

https://doi.org/10.1016/j.eurpolymj.2013.02.019

12. Wei, Z., Zhang, Z., Wan, M. Formation Mechanism of Selfassembled Polyaniline Micro/nanotubes Langmuir 18 2002: pp. $917-921$. https://doi.org/10.1021/la0155799

13. Hsieh, B.Z., Chuang, H.Y., Chao, L. Formation Mechanism of Nanotubular Polyanilines Prepared by an Emulsion Polymerization without Organic Solvent Polymer 49 2008: pp. $4218-4225$.

https://doi.org/10.1016/j.polymer.2008.07.008

14. Shi, L.J. Effect of Structural Changes on The Aggregation Behavior of Imidazolium Ionic Liquids. Shandong: Shandong University 2013.

15. Arkhipova, D., Ermolaev, V., Miluykov, V. Functionalized Phosphonium Ionic Liquids: Synthesis and Application Taylor \& Francis 190 (5) 2015: pp. 899-901. https://doi.org/10.1080/10426507.2014.993759

16. Guo, L.Y., Zhang, B., Bai, S. Synthesis and Application of Functionalized Ionic Liquids as Solvent to Corn Stalk for Phenolic Resin Modification E-Polymers 15 (3) 2015: pp. $195-201$. https://doi.org/10.1515/epoly-2014-0195

17. Nandwani, S.K., Malek, N.I. Study on Evaluating Potential of Imidazolium Ionic Liquids as A Surfactant in Enhanced Oil Recovery Colloids and Surfaces A: Physicochemical and Engineering Aspects 516 (5) 2016: pp. 383-393. https://doi.org/10.1016/j.colsurfa.2016.12.037

18. Qi, X.J., Zhang, X.L., Luo, G.X. Mixing Behavior of Conventional Cationic Surfactants and Ionic Liquid Surfactant 1-Tetradecyl-3-methylimidazolium Bromide
([C14mim]Br) in Aqueous Medium Journal of Dispersion Science and Technology 34 (1) 2013: pp. 125-133. https://doi.org/10.1080/01932691.2011.653926

19. Antonietti, M., Kuang, D., Smarsly, B., Zhou, Y. Ionic liquids for The Convenient Synthesis of Functional Nanoparticles and Other Inorganic Nanostructures Angewandte Chemie International Edition 43 2004: pp. $4988-4992$. https://doi.org/10.1002/chin.200447230

20. Modaressi, A., Sifaoui, H., Mielcarz, M. Influence of The Molecular Structure on The Aggregation of Imidazolium Ionic Liquids in Aqueous Solutions Colloid Surface A 302 2007: pp. $181-185$. https://doi.org/10.1016/j.colsurfa.2007.02.020

21. Zhao, M.W. Application of Ionic Liquids in The Construction of Ordered Molecular Aggregates and Their Application in The Preparation of Nanomaterials. Shandong: Shandong University, 2011.

22. Kang, W.P., Dong, B., Gao, Y.A. Aggregation Behavior of Long-chain Imidazolium Ionic Liquids in Ethyl-ammonium Nitrate Springer Journal $288 \quad(12-13)$ 2010: pp. $1225-1232$. https://doi.org/10.1007/s00396-010-2254-2

23. Pahovnik, D., Žagar, E., Vohlidal, J. Ionic Liquid-induced Formation of Polyaniline Nanostructures during The Chemical Polymerization of Aniline in an Acidic Aqueous Medium Synthetic Metals 160 2010: pp. 1761-1766. https://doi.org/10.1016/j.synthmet.2010.06.016

24. Krishna, A., $\quad$ Laslau, C., $\quad$ Waterhouse, G.I.N., Zujovic, Z.D., Travas-Sejdic, J. Effect of Ionic Liquid on Polyaniline Chemically Synthesised under Falling-pH Conditions Original Paper 67 (8) 2013: pp. 995-1001. https://doi.org/10.2478/s11696-013-0327-7

25. Trchová, M., Šeděnková, I., Konyushenko, E.N. Evolution of PANI Nanotubes: Oxidation of Aniline in Water Journal of Physical Chemistry B 110 (19) 2016: pp. 9461 -9468. https://doi.org/10.1021/jp057528g

26. Ran, F, Yang, Y.L., Zhao, L., Niu, X.Q., Zhang, D.J., Kong, L.B., Luo, Y.C., Kang, L. Preparation of NanoPANI@MnO 2 by Surface Initiated Polymerization Method Using as A Nano-tubular Electrode Material: The Amount Effect of Aniline on The Microstructure and Electrochemical Performance Journal of Energy Chemistry 24(4) 2015: pp. $388-393$. https://doi.org/10.1016/j.jechem.2015.07.003

27. Yu, Q.B., Li, X.H., Zhang, L.G., Wang, X.X., Tao, Y.L., Zhang, M.X. Significantly Improving the Performance and Dispersion Morphology of Porous G-C $3 \mathrm{~N}_{4} / \mathrm{PANI}$ Composites by an Interfacial Polymerization Method e-Polymers 15 (2) 2015: pp. $95-101$. https://doi.org/10.1515/epoly-2014-0218 